\section{(a) \\ BRAZIULIAN JOURNAL \\ OF MEDICAL AND BIOLOGICAL RESEARCH}

www.bjournal.com.br
ISSN 0100-879X

Volume 45 (7) 565-680

July 2012

CLINICAL INVESTIGATION

Braz J Med Biol Res, July 2012, Volume 45(7) 632-636

doi: 10.1590/S0100-879X2012007500073

Interleukin-10-dependent down-regulation of interferon-gamma response to Leishmania by Mycobacterium leprae antigens during the clinical course of a coinfection

R.B.G. Azeredo-Coutinho, D.C.S. Matos, J.A.C. Nery, C.M. Valete-Rosalino and

S.C.F. Mendonça

The Brazilian Journal of Medical and Biological Research is partially financed by

\section{Q}

da Ciência e Tecnologia

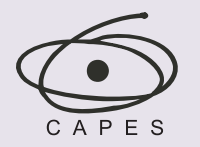

Ministério da Educação

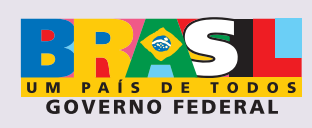

DTFAPESP

Institutional Sponsors

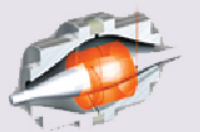

Ф SHIMADZU UNICAMP

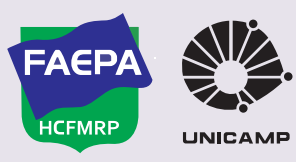

lore High - Performance MS Orbitrap Technology In Proteomics \& Metabolomics analitica Thermo 


\title{
Interleukin-10-dependent down-regulation of interferon-gamma response to Leishmania by Mycobacterium leprae antigens during the clinical course of a coinfection
}

\author{
R.B.G. Azeredo-Coutinho ${ }^{1}$, D.C.S. Matos ${ }^{2}$, J.A.C. Nery ${ }^{3}$, \\ C.M. Valete-Rosalino ${ }^{4}$ and S.C.F. Mendonça ${ }^{1}$ \\ ${ }^{1}$ Laboratório de Imunoparasitologia, Instituto Oswaldo Cruz, \\ Fundação Oswaldo Cruz, Rio de Janeiro, RJ, Brasil \\ 2Laboratório de Tecnologia Imunológica, Instituto de Tecnologia em Imunobiológicos, \\ Fundação Oswaldo Cruz, Rio de Janeiro, RJ, Brasil \\ ${ }^{3}$ Centro de Referência Nacional em Hanseníase, Instituto Oswaldo Cruz, \\ Fundação Oswaldo Cruz, Rio de Janeiro, RJ, Brasil \\ ${ }^{4}$ Departamento de Doenças Infecciosas, Instituto de Pesquisa Clínica Evandro Chagas, \\ Fundação Oswaldo Cruz, Rio de Janeiro, RJ, Brasil
}

\begin{abstract}
We have described a case of a patient with an intriguing association of mucocutaneous leishmaniasis with lepromatous leprosy, two opposite polar forms of these spectral diseases. In the present follow-up study, we investigated the effect of the addition of Mycobacterium leprae antigens on interferon-gamma (IFN-y) production in Leishmania antigen-stimulated cultures of peripheral blood mononuclear cells (PBMC) from this patient. For this purpose, PBMC cultures were stimulated with crude $L$. braziliensis and/or M. leprae whole-cell antigen extracts or with concanavalin A. In some experiments, neutralizing anti-human interleukin (IL)-10 antibodies were added to the cultures. IFN- $\gamma$ and IL-10 levels in culture supernatants were measured by ELISA. During active leprosy, $M$. leprae antigens induced $72.3 \%$ suppression of the IFN- $\gamma$ response to $L$. braziliensis antigen, and this suppression was abolished by IL-10 neutralization. Interestingly, the suppressive effect of $M$. leprae antigen was lost after the cure of leprosy and the disappearance of this effect was accompanied by exacerbation of mucosal leishmaniasis. Considered together, these results provide evidence that the concomitant lepromatous leprosy induced an IL-10-mediated regulatory response that controlled the immunopathology of mucosal leishmaniasis, demonstrating that, in the context of this coinfection, the specific immune response to one pathogen can influence the immune response to the other pathogen and the clinical course of the disease caused by it. Our findings may contribute to a better understanding of the Leishmania/M. leprae coinfection and of the immunopathogenesis of mucosal leishmaniasis.
\end{abstract}

Key words: Interferon-gamma; Interleukin-10; Mucocutaneous leishmaniasis; Lepromatous leprosy; Leishmania braziliensis; Mycobacterium leprae

\section{Introduction}

Leprosy and American tegumentary leishmaniasis are both caused by intracellular organisms. These diseases exhibit similar spectra of clinical forms, which are dependent on antigen-specific cell-mediated immunity. Diffuse cutaneous leishmaniasis and lepromatous leprosy are considered to be the anergic pole. These clinical forms have been associated with high expression of interleukin (IL)-10 and low expression of interferon (IFN)-y $(1,2)$. At the other end of the spectrum, mucosal leishmaniasis and tuberculoid leprosy are associated with a conspicuous cell-mediated immune response in which proinflammatory cytokines, such as IFN- $y$, predominate $(3,4)$. Although the immunopathogenesis of these diseases has been extensively investigated, there are very few immunological studies on leishmaniasis/lep-

Correspondence: R.B.G. Azeredo-Coutinho, Laboratório de Imunoparasitologia, Instituto Oswaldo Cruz, Fiocruz, Av. Brasil, 4365, 21045-900 Rio de Janeiro, RJ, Brasil. Fax: +55-21-2290-0479. E-mail: coutinhob@ymail.com

Received October 17, 2011. Accepted March 14, 2012. Available online May 11, 2012. Published July 2, 2012. 
rosy coinfection, especially in HIV-negative patients $(5,6)$. We have previously described an unusual association of mucosal leishmaniasis and lepromatous leprosy, opposite clinical forms in the immunopathological spectra of these diseases, and have characterized the cytokine response to Leishmania and Mycobacterium leprae antigens during coinfection. Although the stimulation of peripheral blood mononuclear cells (PBMC) with Leishmania and M. leprae antigens induced comparable levels of tumor necrosis factor (TNF)- $\alpha$, IL-5 and IL-10, the IFN- $\gamma$ response to Leishmania antigens was extremely high while the response to $M$. leprae antigens was nearly absent (6). The contrasting responses to Leishmania and $M$. leprae antigens seen in this patient, leading to divergent polar forms of leishmaniasis and leprosy, prompted us to investigate a possible effect of the immune response to one infection on the course of the other. Thus, we evaluated the effect of the addition of $M$. leprae antigens on IFN-Y production in $L$. braziliensis antigen-stimulated PBMC cultures.

\section{Material and Methods}

\section{Case presentation}

A 46-year-old male patient presented with a clinically active mucosal leishmaniasis and lepromatous leprosy coinfection, the clinical picture of which has been previously described in detail (6). The diagnosis of polar lepromatous leprosy was based on positive results from smears of skin samples from the earlobes, elbows, and knees, with an average bacterial index of 4+ with Ziehl-Nielsen staining. Mucosal leishmaniasis was diagnosed by isolation of Leishmania from the nasal mucosal lesion. The isolated parasites were characterized as Leishmania braziliensis by isoenzyme analysis (7). The patient was treated for leishmaniasis with meglumine antimoniate for 30 days with a low-dose schedule of $5 \mathrm{mg} / \mathrm{kg}$ per day of pentavalent antimony and for leprosy with a multidrug therapy regimen (6) with 24 monthly supervised doses. Leprosy treatment was started 10 months before the beginning of leishmaniasis therapy and ended 13 months after its completion. Nine months after diagnosis, the leishmaniasis lesion was considered to be healed, leaving partial mutilation of the nose. Relapse was noticed 13 months after cure of the mucosal lesion, with erosion of the nose and large edema and infiltration of the nose and lips extending to the malar region. On that occasion, Leishmania parasites were again isolated from a biopsy specimen from the nasal lesion, but the patient had no clinical sign of leprosy and skin smears obtained bilaterally from earlobes, elbows, and knees showed a bacterial index of 0 (zero).

\section{Methods}

Venous blood samples were obtained twice before and twice after the cure of leprosy. The patient was clinically cured of mucosal leishmaniasis when the experiments were performed before the cure of leprosy. PBMC were obtained, cultured and stimulated with crude L. braziliensis and/or M. leprae whole-cell antigen extracts $(50 \mathrm{mg} / \mathrm{mL})$ or with concanavalin A (ConA) $(20 \mathrm{mg} / \mathrm{mL})$. The antigen extracts were prepared as previously described (6). Briefly, L. braziliensis (WHO reference strain MHOM/BR/75/2903) promastigotes were separately cultured in Novy-McNealNicolle medium (8), supplemented with RPMI 1640 medium (Sigma, USA) and 10\% heat-inactivated fetal calf serum (Sigma). Stationary phase promastigotes were washed three times by centrifugation at $900 \mathrm{~g}$ for $10 \mathrm{~min}$ at $4^{\circ} \mathrm{C}$ in PBS and were disrupted by 10 repeated cycles of freezing and thawing, followed by ultrasonication (Ultra-tip Labsonic System; Lab-Line, USA) at 40 watts for $15 \mathrm{~min}$ in an ice bath. An irradiated, armadillo-derived whole M. leprae antigen preparation $\left(2 \times 10^{9}\right.$ bacteria/mg; kindly provided by Dr. M.C. Pessolani, Laboratório de Hanseníase, Oswaldo Cruz Foundation, Rio de Janeiro, Brazil) was sonicated according to the same protocol as used for the Leishmania antigen extracts. Previous publications have shown that the antigen extracts used in this study stimulate production of TNF- $\alpha$ and IL-10 in PBMC cultures from healthy subjects (9-11). The antigen concentration in each preparation was adjusted to $1 \mathrm{mg} / \mathrm{mL}$ protein nitrogen. All samples were kept at $-20^{\circ} \mathrm{C}$ until use.

Neutralizing anti-human IL-10 antibodies (BD Pharmingen, USA) were added to some cultures at a final concentration of $50 \mu \mathrm{g} / \mathrm{mL}$, concomitantly with the antigens. IFN- $y$ and IL-10 levels in PBMC culture supernatants were estimated by ELISA as previously described (6). The results are reported as the differences between the mean cytokine levels in stimulated and unstimulated (with medium alone) triplicate cultures. The detection limit of the assays was 8 $\mathrm{pg} / \mathrm{mL}$ for both IFN-y and IL-10. This research protocol was carried out in compliance with the Helsinki Declaration, and was approved by the Ethics Review Committee of Instituto de Pesquisa Clínica Evandro Chagas, Fundação Oswaldo Cruz, Brazilian Ministry of Health, under the reference No. CAAE 0016.0.009.000-02.

\section{Results}

Figure 1 shows the results of representative experiments performed before (Figure 1A and C) and after (Figure 1B and D) the cure of leprosy. On both occasions, the patient's PBMC produced high levels of IFN-y in response to the $L$. braziliensis antigen extract and no IFN- $\gamma$ response when stimulated with $M$. leprae antigens (Figure $1 \mathrm{~A}$ and $\mathrm{B}$ ). The neutralization of IL-10 (Figure 1C and D) did not reverse the inability of the patient's cells to secrete IFN- $\gamma$ in response to $M$. leprae (Figure $1 \mathrm{~A}$ and $\mathrm{B}$ ). The levels of IFN-Y and IL-10 in unstimulated cultures were 0 (Figure 1A), 39.3 $\mathrm{pg} / \mathrm{mL}$ (Figure 1B), $130.9 \mathrm{pg} / \mathrm{mL}$ (Figure 1C), and $31.2 \mathrm{pg} /$ $\mathrm{mL}$ (Figure 1D).

We investigated whether the $M$. leprae antigens could 
inhibit the IFN-Y production stimulated by $L$. braziliensis antigens. This was indeed the case in the two experiments performed before the cure of leprosy, when cultures stimulated with both antigen preparations produced less than one third of the amount of IFN-y measured in supernatants of cultures stimulated with $L$. braziliensis antigens alone. Figure $1 \mathrm{~A}$ shows the results of one of these experiments. In an experiment performed only once, the $M$. leprae antigens were unable to inhibit the production of IFN-y induced by ConA, and the addition of neutralizing anti-IL-10 antibodies had no effect on the IFN-y levels in this particular experiment (data not shown).

The $M$. leprae antigen-induced suppression of the IFN-y response to the $L$. braziliensis extract observed before the cure of leprosy was blocked by neutralizing anti-IL-10 antibodies (Figure 1A). Interestingly enough, the suppressive effect of $M$. leprae antigens in the $L$. braziliensis antigen-induced IFN-Y production could not be seen after the cure of leprosy, when the cultures stimulated with both antigen preparations produced as much IFN-y as the cultures stimulated with $L$. braziliensis antigens alone. Figure $1 \mathrm{~B}$ shows the results of one of two experiments with similar results performed after the cure of leprosy. The loss of the $M$. leprae antigen-induced suppression of the IFN-y response to $L$. braziliensis antigens observed in vitro was clinically accompanied by the reactivation and exacerbation of mucosal leishmaniasis (Figure 2).

\section{Discussion}

The high production of IFN-y after stimulation with $L$. braziliensis antigens and the absence of this cytokine after stimulation with $M$. leprae antigens observed both before and after the cure of leprosy are in accordance with previous results obtained with this patient (6). The absence of an IFN- $\gamma$ response to the $M$. leprae antigen extract was not due to IL-10-mediated suppression since neutralization of IL-10 did not change the inability of the patient's cells to produce IFN-y in response to $M$. leprae antigens, as previously reported (6). During active leprosy, $M$. leprae antigens were able to markedly inhibit the IFN-y production in Leishmania antigen-stimulated PBMC cultures, which was restored by the addition of neutralizing anti-IL-10 antibodies, indicating that this cytokine plays a role in the observed immunosuppression. The down-regulatory effect of the $M$. leprae antigens on the IFN-y response induced by Leishmania antigens was completely lost after the cure of leprosy, and the loss of this effect was accompanied

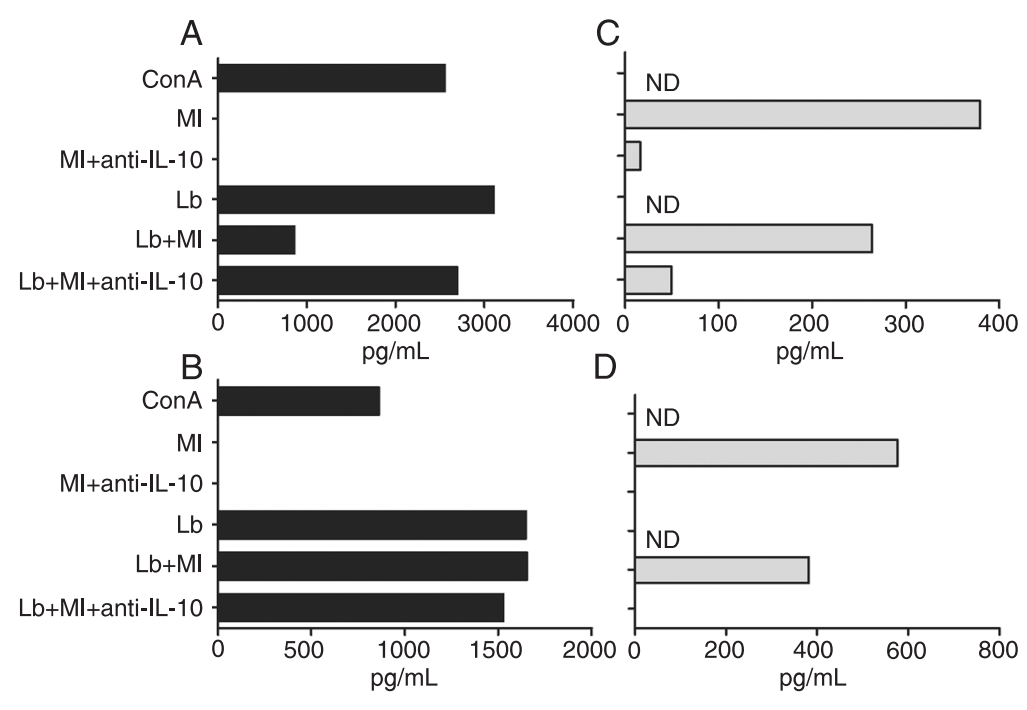

Figure 1. IFN-y and IL-10 levels in PBMC culture supernatants estimated by ELISA. A, B, IFN- $y$ levels in PBMC cultures in the presence of Mycobacterium leprae and Leishmania braziliensis whole antigen extracts (Ml and Lb, respectively), concanavalin A (ConA), and neutralizing anti-IL-10 antibody (anti-IL-10), alone or in combination. $C, D, \mathrm{IL}-10$ levels in cultures stimulated with $\mathrm{Ml}$ alone or with $\mathrm{Lb}$, in the presence or absence of anti-IL-10. $A, C$, During active leprosy; $B, D$, after cure of leprosy. The results shown represent the differences between the levels obtained in stimulated and unstimulated cultures (with medium alone). IFN- $\gamma=$ interferon- $y$; IL-10 = interleukin-10; PBMC = peripheral blood mononuclear cells; $\mathrm{ND}=$ not done.
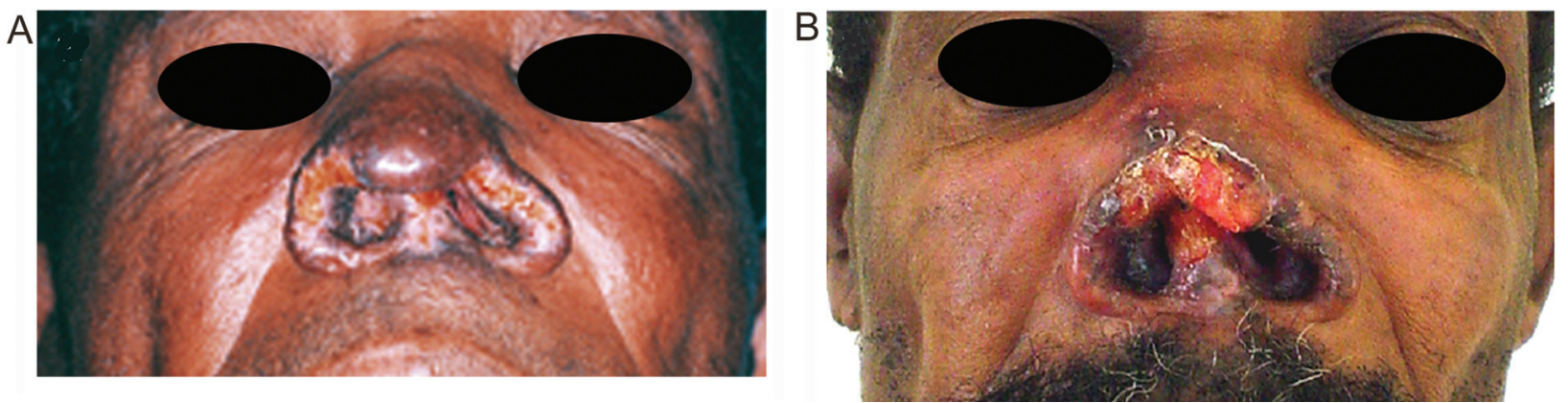

Figure 2. Clinical appearance of the leishmaniasis mucosal lesion. $A$, During active leprosy. $B$, Exacerbation after the cure of leprosy. 
by worsening of mucosal leishmaniasis lesions. Besides IL-10, other factors and cells such as IL-4, indoleamine 2, 3-dioxygenase and CD8 ${ }^{+} \mathrm{T}$ cells have also been suggested to participate in the specific T-cell anergy found in patients with lepromatous leprosy $(2,12,13)$. Although we cannot rule out the participation of other suppressive mechanisms that could be absent after the healing of leprosy, it is possible that the exacerbation of mucosal leishmaniasis was responsible for the lack of responsiveness to the IL-10-induced suppression of IFN-y production. It has been reported that PBMC from mucosal leishmaniasis patients are poorly responsive to IL-10-mediated suppression of IFN-y production (14). It should be noted that, in contrast to the reactivation of the mucosal lesion seen when the experiments were performed after the cure of leprosy, the patient was clinically cured of mucosal leishmaniasis when the previous experiments were performed before the cure of leprosy.

Taken together, these observations indicate that coinfection with $M$. leprae interfered with the course of mucosal leishmaniasis. Lepromatous leprosy is characterized by the absence of specific cellular immunity to $M$. leprae, resulting in uncontrolled proliferation of bacilli (12). A number of factors have been implicated as causes of the specific T-cell anergy found in patients with lepromatous leprosy, including $\mathrm{CD}^{+}$and regulatory T cells, IL-4 and IL-10 $(2,3,12,15)$. However, we have found that neutralization of IL-10 did not increase the production of IFN- $y$ in $M$. leprae antigenstimulated cultures of PBMCs from this multibacillary patient, indicating that the lack of IFN-y production by the patient's T cells was not due to IL-10-mediated suppression. Mucosal leishmaniasis patients have up-regulated IFN- $\gamma$ and TNF- $\alpha$ secretion and decreased IL-10 secretion when compared to patients with uncomplicated cutaneous leishmaniasis (4). It has been shown that mucosal leishmaniasis lesions possess a higher number of IFN- $y$-producing cells compared to cutaneous leishmaniasis lesions. Here and in a previously published article (6) we showed that the IFN-y response of this patient to Leishmania antigens was remarkably high, as expected in mucosal leishmaniasis patients. It was even higher than the response obtained with polyclonal stimulation using ConA (Figure $1 \mathrm{~A}$ and $1 \mathrm{~B}$ ). This result agrees with the data shown in our previous study with this patient, in which the levels of proinflammatory cytokines (IFN- $\gamma$ and TNF- $\alpha$ ) were higher with Leishmania antigen stimulation than with ConA stimulation (6).

Moreover, a decreased in situ expression of the IL10 receptor was proposed as a possible mechanism for the exacerbated inflammatory and cytotoxic responses

\section{References}

1. Akuffo $H$, Maasho $K$, Blostedt $M$, Hojeberg $B$, Britton $S$, Bakhiet M. Leishmania aethiopica derived from diffuse leishmaniasis patients preferentially induce mRNA for inter- involved in tissue damage in the mucosal lesions caused by $L$. braziliensis infection (16). IL-10 is a potent inhibitor of the production of cytokines involved in the inflammatory response, such as TNF- $\alpha$, IL-1, IFN- $\gamma$, and IL-12 (17). There is solid evidence that live $L$. braziliensis parasites persist after the clinical cure of American tegumentary leishmaniasis (18). Relapse of leishmaniasis mucosal lesions is frequent (19) and is a challenge for clinicians. Recently, local immunological factors associated with recurrence of mucosal leishmaniasis were described using immunohistochemical analysis of cell phenotypes and cytokines present in the inflammatory infiltrate of mucosal lesions. IFN-y-producing cells were detected in active lesions and decreased after therapy (19). On the other hand, IL-10 expression was markedly increased in cured patients. These observations suggest that IL-10 plays a role in the control of pathology in mucosal leishmaniasis.

Most reports on immunomodulation during coinfection refer to associated HIVIAIDS infections. It has also been shown that helminth infections can induce immunomodulation and interfere with the course of several infectious diseases (20), including leprosy (21) and leishmaniasis (22). Interestingly, the influence of this association with helminthic infections may either be beneficial or deleterious to the clinical course of protozoan diseases such as malaria (20).

Studies on the association of leprosy and leishmaniasis are rare, and, to our knowledge, in this coinfection, this is the first report of the influence of one infection (with $M$. leprae) on the immune response to the other pathogen $(L$. braziliensis) and on the clinical course of the disease caused by it (mucosal leishmaniasis). Our results indicate that concomitant leprosy induced an IL-10-mediated regulatory response that might have controlled the immunopathology of mucosal leishmaniasis. It should be remembered, however, that this patient had an unusual association of two opposite polar forms of these spectral diseases: lepromatous leprosy (anergic, multibacillary) and mucosal leishmaniasis (hyperergic, pauciparasitic). The study of other cases of association of leprosy and tegumentary leishmaniasis may increase our knowledge about the interference that one infection can have on the immunopathogenesis and clinical course of the other.

\section{Acknowledgments}

S.C.F. Mendonça is the recipient of a fellowship as a CNPq investigator. leukin-10 while those from localized leishmaniasis patients induce interferon-gamma. J Infect Dis 1997; 175: 737-741.

2. Sieling PA, Abrams JS, Yamamura M, Salgame P, Bloom 
$\mathrm{BR}$, Rea $\mathrm{TH}$, et al. Immunosuppressive roles for IL-10 and IL-4 in human infection. In vitro modulation of $\mathrm{T}$ cell responses in leprosy. J Immunol 1993; 150: 5501-5510.

3. Modlin RL. Th1-Th2 paradigm: insights from leprosy. J Invest Dermatol 1994; 102: 828-832.

4. Bacellar $\mathrm{O}$, Lessa $\mathrm{H}$, Schriefer $\mathrm{A}$, Machado $\mathrm{P}$, Ribeiro de JA, Dutra WO, et al. Up-regulation of Th1-type responses in mucosal leishmaniasis patients. Infect Immun 2002; 70: 6734-6740.

5. Delobel P, Launois P, Djossou F, Sainte-Marie D, Pradinaud R. American cutaneous leishmaniasis, lepromatous leprosy, and pulmonary tuberculosis coinfection with downregulation of the T-helper 1 cell response. Clin Infect Dis 2003; 37: 628633.

6. Matos DS, Azeredo-Coutinho RB, Schubach A, ConceiçãoSilva F, Baptista C, Moreira JS, et al. Differential interferongamma production characterizes the cytokine responses to Leishmania and Mycobacterium leprae antigens in concomitant mucocutaneous leishmaniasis and lepromatous leprosy. Clin Infect Dis 2005; 40: e5-12.

7. Cupolillo E, Grimaldi G Jr, Momen H. A general classification of New World Leishmania using numerical zymotaxonomy. Am J Trop Med Hyg 1994; 50: 296-311.

8. Nicolle C. Culture des corps de Leishman isolés de la rate dans trois cas d'anémie splenique infantile. Bull Soc Pathol Exot 1908; 1: 121-126.

9. Telino E, De Luca PM, Matos DC, Azeredo-Coutinho RB, Meirelles MN, Conceição-Silva $\mathrm{F}$, et al. In vitro responses of human peripheral blood mononuclear cells to whole-cell, particulate and soluble extracts of Leishmania promastigotes. Clin Exp Immunol 2006; 143: 338-344.

10. Matta NE, Nogueira RS, Franco AM, de Souza E Souza, Mattos MS, Oliveira-Neto MP, et al. Leishmania (Viannia) guyanensis induces low immunologic responsiveness in leishmaniasis patients from an endemic area of the Brazilian Amazon Highland. Am J Trop Med Hyg 2009; 80: 339-344.

11. Lima MC, Pereira GM, Rumjanek FD, Gomes HM, Duppre $\mathrm{N}$, Sampaio EP, et al. Immunological cytokine correlates of protective immunity and pathogenesis in leprosy. Scand $J$ Immunol 2000; 51: 419-428.

12. Salgame P, Modlin R, Bloom BR. On the mechanism of human T cell suppression. Int Immunol 1989; 1: 121-129.
13. de Souza Sales J, Lara FA, Amadeu TP, de Oliveira Fulco T, da Costa Nery JA, Sampaio EP, et al. The role of indoleamine 2,3-dioxygenase in lepromatous leprosy immunosuppression. Clin Exp Immunol 2011; 165: 251-263.

14. Carvalho LP, Passos S, Dutra WO, Soto M, Alonso C, Gollob $\mathrm{KJ}$, et al. Effect of LACK and KMP11 on IFN-gamma production by peripheral blood mononuclear cells from cutaneous and mucosal leishmaniasis patients. Scand J Immunol 2005; 61: 337-342.

15. Britton WJ, Lockwood DN. Leprosy. Lancet 2004; 363: 1209 1219.

16. Faria DR, Gollob KJ, Barbosa J Jr, Schriefer A, Machado PR, Lessa $\mathrm{H}$, et al. Decreased in situ expression of interleukin-10 receptor is correlated with the exacerbated inflammatory and cytotoxic responses observed in mucosal leishmaniasis. Infect Immun 2005; 73: 7853-7859.

17. D'Andrea A, Aste-Amezaga M, Valiante NM, Ma X, Kubin M, Trinchieri G. Interleukin 10 (IL-10) inhibits human lymphocyte interferon gamma-production by suppressing natural killer cell stimulatory factor/lL-12 synthesis in accessory cells. J Exp Med 1993; 178: 1041-1048.

18. Schubach A, Cuzzi-Maya T, Oliveira AV, Sartori A, de Oliveira-Neto MP, Mattos MS, et al. Leishmanial antigens in the diagnosis of active lesions and ancient scars of American tegumentary leishmaniasis patients. Mem Inst Oswaldo Cruz 2001; 96: 987-996.

19. Tuon FF, Gomes-Silva A, Da-Cruz AM, Duarte MI, Neto VA, Amato VS. Local immunological factors associated with recurrence of mucosal leishmaniasis. Clin Immunol 2008; 128: $442-446$

20. van Riet E., Hartgers FC, Yazdanbakhsh M. Chronic helminth infections induce immunomodulation: consequences and mechanisms. Immunobiology 2007; 212: 475-490.

21. Diniz LM, Zandonade E, Dietze R, Pereira FE, RibeiroRodrigues R. Short report: do intestinal nematodes increase the risk for multibacillary leprosy? Am J Trop Med Hyg 2001; 65: 852-854.

22. O'Neal SE, Guimarães LH, Machado PR, Alcântara L, Morgan DJ, Passos S, et al. Influence of helminth infections on the clinical course of and immune response to Leishmania braziliensis cutaneous leishmaniasis. J Infect Dis 2007; 195: 142-148. 